(n)

\title{
The number of independent Traces and Supertraces on the Symplectic Reflection Algebra $H_{1, \eta}\left(\Gamma \mathrm{\imath} S_{N}\right)$
}

S.E. Konstein, I.V. Tyutin

To cite this article: S.E. Konstein, I.V. Tyutin (2018) The number of independent Traces and Supertraces on the Symplectic Reflection Algebra $H_{1, \eta}\left(\Gamma \backslash S_{N}\right)$, Journal of Nonlinear Mathematical Physics 25:3, 485-496, DOI:

https://doi.org/10.1080/14029251.2018.1494768

To link to this article: https://doi.org/10.1080/14029251.2018.1494768

Published online: 04 January 2021 


\title{
The number of independent Traces and Supertraces on the Symplectic Reflection Algebra $\left.H_{1, \eta}(\Gamma) S_{N}\right)$
}

\author{
S.E. Konstein \\ I.E. Tamm Department of Theoretical Physics, \\ P.N. Lebedev Physical Institute, 53, Leninsky Prospect \\ Moscow, 117924, Russia \\ konstein@lpi.ru \\ I.V. Tyutin \\ I.E. Tamm Department of Theoretical Physics, \\ P.N. Lebedev Physical Institute, 53, Leninsky Prospect \\ Moscow, 117924, Russia \\ Tomsk State Pedagogical University, Kievskaya St. 60, 634061 Tomsk, Russia \\ tyutin@lpi.ru
}

Received 28 November 2017

Accepted 27 March 2018

\begin{abstract}
Symplectic reflection algebra $H_{1, \eta}(G)$ has a $T(G)$-dimensional space of traces whereas, when considered as a superalgebra with a natural parity, it has an $S(G)$-dimensional space of supertraces. The values of $T(G)$ and $S(G)$ depend on the symplectic reflection group $G$ and do not depend on the parameter $\eta$.

In this paper, the values $T(G)$ and $S(G)$ are explicitly calculated for the groups $G=\Gamma$ ? $S_{N}$, where $\Gamma$ is a finite subgroup of $\operatorname{Sp}(2, \mathbb{C})$.
\end{abstract}

\section{Introduction}

Let $V:=\mathbb{C}^{2 N}$, let $G \subset S p(2 N, \mathbb{C})$ be a finite group generated by symplectic reflections. In [11], it was shown that Symplectic Reflection Algebra $H_{1, \eta}(G)$ has $T(G)$ independent traces, where $T(G)$ is the number of conjugacy classes of elements without eigenvalue 1 belonging to the group $G \subset$ $S p(2 N) \subset \operatorname{End}(V)$, and that the algebra $H_{1, \eta}(G)$, considered as a superalgebra with a natural parity, has $S(G)$ independent supertraces, where $S(G)$ is the number of conjugacy classes of elements without eigenvalue -1 belonging to $G \subset S p(2 N) \subset \operatorname{End}(V)$. Hereafter, speaking about spectrum, eigenvalues and eigenvectors, the rank of an element of the group algebra $\mathbb{C}[G]$ of the group $G$, etc., we have in mind the representation of the group algebra $\mathbb{C}[G]$ in the space $V$. Besides, we denote all the units in groups, algebras, etc., by 1 , and $c \cdot 1$ by $c$ for any number $c$.

Apart from a few cases, there are two families of groups generated by symplectic reflections, see [7] and also [9], [2], [5]:

Family 1): $G$ is a complex reflection group acting on $\mathfrak{H} \oplus \mathfrak{H}^{*}$, where $\mathfrak{H}$ is the space of reflection representation. In this case, $G$ is a direct product of several groups from the following set of Coxeter groups

$$
A_{n}(n \geq 1), B_{n}=C_{n}(n \geq 2), D_{n}(n \geq 3), E_{6}, E_{7}, E_{8}, F_{4}, G_{2}, H_{3}, H_{4}, I_{2}(n)(n \geq 5, n \neq 6) .
$$

Family 2): $G=\Gamma$ ( $S_{N}$, which means here $G=\Gamma^{N} \rtimes S_{N}$ acting on $\left(\mathbb{C}^{2}\right)^{N}$, where $\Gamma$ is a finite subgroup of $\operatorname{Sp}(2, \mathbb{C})$. 
For groups $G$ from the set (1.1), the list of values $T(G)$ and $S(G)$ is given in [10].

In this work, we give the values of $T(G)$ and $S(G)$ for the 2nd family. Namely, we found the generating functions

$$
\begin{aligned}
t(\Gamma, x) & :=\sum_{N=0}^{\infty} T\left(\Gamma \imath S_{N}\right) x^{N}, \\
s(\Gamma, x) & :=\sum_{N=0}^{\infty} S\left(\Gamma \imath S_{N}\right) x^{N}
\end{aligned}
$$

for each finite subgroup $\Gamma \subset S p(2, \mathbb{C})$, see Theorem 5.1.

All needed definitions are given in the Section 2; the structure, conjugacy classes and characteristic polynomials of the groups $\Gamma$ ? $S_{N}$ are described in Section 3.

To include the case $N=0$ in consideration in formulas (1.2)-(1.3), it is natural to set $\Gamma$ ? $S_{0}:=$ $\{E\}$ and, since $\operatorname{dim} V=0$, to set $H_{1, \eta}\left(\Gamma \succ S_{0}\right):=\mathbb{C}[\{E\}]$, where $\{E\}$ is the group containing only one element $E$.

Applying the definitions given in Section 2 to the algebra $\left.H_{1, \eta}(\Gamma) S_{0}\right)$ we deduce that

a) if the algebra $H_{1, \eta}\left(\Gamma \succ S_{0}\right)=\mathbb{C}[\{E\}]$ is considered as superalgebra, it has only a trivial parity $\pi \equiv 0$

b) the algebra $H_{1, \eta}\left(\Gamma \imath S_{0}\right)=\mathbb{C}[\{E\}]$ has 1-dimensional space of traces and 1-dimensional space of supertraces; these spaces coincide;

c) it is natural to set $\left.T(\Gamma) S_{0}\right)=S\left(\Gamma \nmid S_{0}\right)=1$;

d) the algebra $H_{1, \eta}\left(\Gamma\left\{S_{0}\right)=\mathbb{C}[\{E\}]\right.$ contains two Klein operators (i.e., elements satisfying conditions (2.1)-(2.3)), namely, 1 and -1 .

\section{Preliminaries}

\subsection{Traces}

Let $\mathscr{A}$ be an associative superalgebra with parity $\pi$. All expressions of linear algebra are given for homogenous elements only and are supposed to be extended to inhomogeneous elements via linearity.

A linear complex-valued function str on $\mathscr{A}$ is called a supertrace, if

$$
\operatorname{str}(f g)=(-1)^{\pi(f) \pi(g)} \operatorname{str}(g f) \text { for all } f, g \in \mathscr{A} .
$$

A linear complex-valued function tr on $\mathscr{A}$ is called a trace, if

$$
\operatorname{tr}(f g)=\operatorname{tr}(g f) \text { for all } f, g \in \mathscr{A} .
$$

The element $K \in \mathscr{A}$ is called a Klein operator, if

$$
\begin{aligned}
& \pi(K)=0, \\
& K^{2}=1, \\
& K f=(-1)^{\pi(f)} f K \quad \text { for all } f \in \mathscr{A} .
\end{aligned}
$$

Any Klein operator, if exists, establishes an isomorphism between the space of traces on $\mathscr{A}$ and the space of supertraces on $\mathscr{A}$. 
Namely, if $f \mapsto \operatorname{tr}(f)$ is a trace, then $f \mapsto \operatorname{tr}\left(f K^{1+\pi(f)}\right)$ is a supertrace, and if $f \mapsto \operatorname{str}(f)$ is a supertrace, then $f \mapsto \operatorname{str}\left(f K^{1+\pi(f)}\right)$ is a trace.

\subsection{Symplectic reflection group}

Let $V=\mathbb{C}^{2 N}$ be endowed with a non-degenerate anti-symmetric $\operatorname{Sp}(2 N)$-invariant bilinear form $\omega(\cdot, \cdot)$, let the vectors $e_{i} \in V$, where $i=1 \ldots, 2 N$, constitute a basis in $V$.

The matrix $\left(\omega_{i j}\right):=\omega\left(e_{i}, e_{j}\right)$ is anti-symmetric and non-degenerate.

Let $x^{i}$ be the coordinates of $x \in V$, i.e., $x=e_{i} x^{i}$. Then $\omega(x, y)=\omega_{i j} x^{i} y^{j}$ for any $x, y \in V$. The indices $i$ are lowered and raised by means of the forms $\left(\omega_{i j}\right)$ and $\left(\omega^{i j}\right)$, where $\omega_{i j} \omega^{k j}=\delta_{i}^{k}$.

Definition 2.1. The element $R \in \operatorname{Sp}(2 N) \subset \operatorname{End} V$ is called a symplectic reflection, if $\operatorname{rank}(R-1)=2$.

Definition 2.2. Any finite subgroup $G$ of $\operatorname{Sp}(2 N)$ generated by a set of symplectic reflections is called a symplectic reflection group.

In what follows, $G$ stands for a symplectic reflection group, and $\mathscr{R}$ stands for the set of all symplectic reflections in $G$.

Let $R \in \mathscr{R}$. Set

$$
\begin{aligned}
V_{R} & :=\operatorname{Im}(R-1), \\
Z_{R} & :=\operatorname{Ker}(R-1) .
\end{aligned}
$$

Clearly, $V_{R}$ and $Z_{R}$ are symplectically perpendicular, i.e., $\omega\left(V_{R}, Z_{R}\right)=0$, and $V=V_{R} \oplus Z_{R}$.

So, let $x=x_{V_{R}}+x_{Z_{R}}$ for any $x \in V$, where $x_{V_{R}} \in V_{R}$ and $x_{Z_{R}} \in Z_{R}$. Set

$$
\omega_{R}(x, y):=\omega\left(x_{V_{R}}, y_{V_{R}}\right) \text {. }
$$

\subsection{Symplectic reflection algebra (following [3])}

Let $\mathbb{C}[G]$ be the group algebra of $G$, i.e., the set of all linear combinations $\sum_{g \in G} \alpha_{g} \bar{g}$, where $\alpha_{g} \in \mathbb{C}$.

If we were rigorists, we would write $\bar{g}$ to distinguish $g$ considered as an element of $G \subset \operatorname{End}(V)$ from the same element $\bar{g} \in \mathbb{C}[G]$ considered as an element of the group algebra. The addition in $\mathbb{C}[G]$ is defined as follows:

$$
\sum_{g \in G} \alpha_{g} \bar{g}+\sum_{g \in G} \beta_{g} \bar{g}=\sum_{g \in G}\left(\alpha_{g}+\beta_{g}\right) \bar{g}
$$

and the multiplication is defined by setting $\overline{g_{1}} \overline{g_{2}}=\overline{g_{1} g_{2}}$. In what follows, however, we abuse notation and omit the bar sign over elements of the group algebra.

Let $\eta$ be a function on $\mathscr{R}$, i.e., a set of constants $\eta_{R}$ with $R \in \mathscr{R}$ such that $\eta_{R_{1}}=\eta_{R_{2}}$, if $R_{1}$ and $R_{2}$ belong to one conjugacy class of $G$.

Definition 2.3. The algebra $H_{t, \eta}(G)$, where $t \in \mathbb{C}$, is an associative algebra with unit 1 ; it is the algebra $\mathbb{C}[V]$ of (noncommutative) polynomials in the elements of $V$ with coefficients in the group algebra $\mathbb{C}[G]$ subject to the relations

$$
\begin{aligned}
g x & =g(x) g \text { for any } g \in G \text { and } x \in V, \text { where } g(x)=e_{i} g_{j}^{i} x^{j} \text { for } x=e_{i} x^{i}, \\
{[x, y] } & =t \omega(x, y)+\sum_{R \in \mathscr{R}} \eta_{R} \omega_{R}(x, y) R \text { for any } x, y \in V .
\end{aligned}
$$

The algebra $H_{t, \eta}(G)$ is called a symplectic reflection algebra, see [3]. 
The commutation relations (2.8) suggest to define the parity $\pi$ by setting:

$$
\pi(x)=1, \pi(g)=0 \text { for any } x \in V, \text { and } g \in G,
$$

enabling one to consider $H_{t, \eta}(G)$ as an associative superalgebra.

We consider the case $t \neq 0$ only, for any such $t$ it is equivalent to the case $t=1$.

Let $\mathscr{A}$ and $\mathscr{B}$ be superalgebras such that $\mathscr{A}$ is a $\mathscr{B}$-module. We say that the superalgebra $\mathscr{A} * \mathscr{B}$ is a crossed product of $\mathscr{A}$ and $\mathscr{B}$, if $\mathscr{A} * \mathscr{B}=\mathscr{A} \otimes \mathscr{B}$ as a superspace and

$$
\left(a_{1} \otimes b_{1}\right) *\left(a_{2} \otimes b_{2}\right)=a_{1} b_{1}\left(a_{2}\right) \otimes b_{1} b_{2},
$$

see [14]. The element $b_{1}\left(a_{2}\right)$ may include a sign factor imposed by the Sign Rule, see [1], p. 45.

The (super)algebra $H_{1, \eta}(G)$ is a deform of the crossed product of the Weyl algebra $W_{N}$ and the group algebra of a finite subgroup $G \subset \operatorname{Sp}(2 N)$ generated by symplectic reflections.

\subsection{The number of independent traces and supertraces on the symplectic reflection algebras}

Theorem 2.1 ([11]). Let the symplectic reflection group $G \subset \operatorname{End}(V)$ have $T_{G}$ conjugacy classes without eigenvalue 1 and $S_{G}$ conjugacy classes without eigenvalue -1 .

Then the algebra $H_{1, \eta}(G)$ has $T(G)=T_{G}$ independent traces whereas $H_{1, \eta}(G)$ considered as a superalgebra, see (2.9), has $S(G)=S_{G}$ independent supertraces.

Proposition 2.1. Let $G_{1} \subset \operatorname{End}\left(V_{1}\right), G_{2} \subset \operatorname{End}\left(V_{2}\right)$ and $G=G_{1} \times G_{2} \subset \operatorname{End}\left(V_{1} \oplus V_{2}\right)$ be symplectic reflection groups. Then $T(G)=T\left(G_{1}\right) T\left(G_{2}\right)$ and $S(G)=S\left(G_{1}\right) S\left(G_{2}\right)$.

Proof follows from evident relations $T_{G}=T_{G_{1}} T_{G_{2}}, S_{G}=S_{G_{1}} S_{G_{2}}$ and Theorem 2.1.

Proposition 2.2. If there exists a $K \in G$ such that $\left.K\right|_{V}=-1$, then $K$ is a Klein operator.

\section{The group $\Gamma \geq S_{N}$}

\subsection{Finite subgroups of $\mathrm{Sp}(2, \mathbb{C})$}

The complete list of the finite subgroups $\Gamma \subset \operatorname{Sp}(2, \mathbb{C})$ is as follows, see, e.g., [15]:

\begin{tabular}{|c|c|c|c|}
\hline$\Gamma$ & Order & Presence of -1 & $\begin{array}{c}\text { The number of } \\
\text { conjugacy classes } C(\Gamma)\end{array}$ \\
\hline Cyclic group $\mathbf{Z}_{n}:=\mathbb{Z} / n \mathbb{Z}$ & $n$ & $\begin{array}{l}\text { yes, if } n \text { is even; } \\
\text { no, if } n \text { is odd }\end{array}$ & $n$ \\
\hline Binary dihedral group $\mathscr{D}_{n}$ & $4 n$ & yes & $n+3$ \\
\hline Binary tetrahedral group $\mathscr{T}$ & 24 & yes & 8 \\
\hline Binary octahedral group $\mathscr{O}$ & 48 & yes & 9 \\
\hline Binary icosahedral group $\mathscr{I}$ & 120 & yes & 7 \\
\hline
\end{tabular}

It is easy to see that each of these groups, except $\mathbf{Z}_{2 k+1}$, has $C(\Gamma)-1$ conjugacy classes without +1 in the spectrum and has $C(\Gamma)-1$ conjugacy classes without -1 in the spectrum. The group $\mathbf{Z}_{2 k+1}$ has $C\left(\mathbf{Z}_{2 k+1}\right)-1$ conjugacy classes without +1 in the spectrum and it has $C\left(\mathbf{Z}_{2 k+1}\right)$ conjugacy classes without -1 in the spectrum. 


\subsection{Symplectic reflections in $\Gamma \geq S_{N}$ (following [4])}

Let $V=\mathbb{C}^{2 N}$ and let the symplectic form $\omega$ have the shape

$$
\omega:=\left(\begin{array}{cccc}
\varpi & & & \\
& \varpi & & \\
& & \ddots & \\
& & & \varpi
\end{array}\right) \text {, where } \varpi=\left(\begin{array}{cc}
0 & 1 \\
-1 & 0
\end{array}\right) .
$$

The elements of the group $\Gamma$ ? $S_{N}$ have the form of $N \times N$ block matrix with $2 \times 2$ blocks. Consider the following elements of $\Gamma$ ? $S_{N}$

$$
\begin{aligned}
\left(D_{g, i}\right)_{k l} & :=\left\{\begin{array}{l}
g, \text { if } k=l=i, \\
1, \text { if } k=l \neq i, \\
0, \text { otherwise, }
\end{array}\right. \\
\left(K_{i j}\right)_{k l}: & = \begin{cases}\delta_{k l}, & \text { if } k, l \neq i, k, l \neq j, \\
\delta_{k i} \delta_{l j}+\delta_{k j} \delta_{l i}, & \text { otherwise, }\end{cases} \\
S_{g, i j} & :=D_{g, i} D_{g^{-1}, j} K_{i j},
\end{aligned}
$$

where $i, j=1, \ldots, N, i \neq j, 1 \neq g \in \Gamma$. It is clear that $K_{i j}=K_{j i}$ and $S_{g, i j}=S_{g^{-1}, j i}$.

The complete set of symplectic reflections in $\Gamma$ ? $S_{N}$ consists of $D_{g, i}, K_{i j}$ and $S_{g, i j}$, where $1 \leqslant i<$ $j \leqslant N$ and $1 \neq g \in \Gamma$. This set generates the group $\Gamma$ $S_{N}$.

The symplectic reflections $K_{i j}$ and $S_{g, i j}$ lie in one conjugacy class for all $i \neq j$ and $g \neq 1$; the elements $D_{g, i}(g \neq 1)$ and $D_{h, j}(h \neq 1)$ lie in one conjugacy class, if $g$ and $h$ are conjugate in $\Gamma$. So, the algebra $H_{1, \eta}\left(\Gamma<S_{N}\right)$ depends on $C(\Gamma)$ parameters $\eta$, if $N \geqslant 2$, and on $C(\Gamma)-1$ parameters, if $N=1$. Here $C(\Gamma)$ is the number of conjugacy classes in $\Gamma$ including the class $\{1\}$.

\subsection{Conjugacy classes (following [13])}

Further, the elements of the group $\Gamma \nmid S_{N}$ can be represented in the form $D \sigma$ where $D \in \Gamma^{N}$ is a diagonal $N \times N$ block matrix, each block being a $2 \times 2$-matrix, and $\sigma$ is $N \times N$ block matrix of permutation each block being a $2 \times 2$-matrix.

The product has the form:

$$
\left(D_{1} \sigma_{1}\right)\left(D_{2} \sigma_{2}\right)=D_{3} \sigma_{3}
$$

where $\sigma_{3}=\sigma_{1} \sigma_{2}$ and $D_{3}=D_{1} \sigma_{1} D_{2} \sigma_{1}^{-1}$. 
S.E. Konstein and I.V. Tyutin / The number of independent Traces and Supertraces on the Symplectic Reflection Algebra $H_{1, \eta}\left(\Gamma{ } S_{N}\right)$

Fix an element $g_{0}=D_{0} \sigma_{0}$. Since the permutation $\sigma_{0}$ is a product of cycles, there exists a permutation $\sigma^{\prime}$ such that

$$
\begin{aligned}
& \sigma^{\prime} \sigma_{0}\left(\sigma^{\prime}\right)^{-1}=\left(\begin{array}{cccc}
c_{1} & & & \\
& c_{2} & & \\
& & \ddots & \\
& & & c_{s}
\end{array}\right) \text {, where } c_{k} \text { are the cycles of length } L_{k}, \sum_{k} L_{k}=N, \\
& c_{k}=\left(\begin{array}{cccccc}
0 & 1 & 0 & 0 & \cdots & 0 \\
0 & 0 & 1 & 0 & \cdots & 0 \\
0 & 0 & 0 & 1 & \cdots & 0 \\
\vdots & \vdots & \vdots & \ddots & \ddots & \vdots \\
0 & 0 & 0 & 0 & \cdots & 1 \\
1 & 0 & 0 & 0 & \cdots & 0
\end{array}\right) .
\end{aligned}
$$

The element $\sigma^{\prime} D_{0} \sigma_{0}\left(\sigma^{\prime}\right)^{-1}$ has the form

$$
\sigma^{\prime} D_{0} \sigma_{0}\left(\sigma^{\prime}\right)^{-1}=\left(\begin{array}{llll}
D_{1} c_{1} & & & \\
& D_{2} c_{2} & & \\
& & \ddots & \\
& & & D_{s} c_{s}
\end{array}\right),
$$

where $D_{k}$ is an $L_{k} \times L_{k}$ diagonal block matrix, each block being a $2 \times 2$-matrix:

$$
D_{k}=\left(\begin{array}{cccc}
g_{1}^{k} & & & \\
& g_{2}^{k} & & \\
& & \ddots & \\
& & & g_{L_{k}}^{k}
\end{array}\right), g_{i}^{k} \in \Gamma .
$$

Next, consider diagonal block matrices $H_{k}=\operatorname{diag}\left(h_{1}^{k}, h_{2}^{k}, \ldots, h_{L_{k}}^{k}\right)$ and the elements

$$
H_{k} D_{k} c_{k} H_{k}^{-1}=\left(\begin{array}{llll}
h_{1}^{k} g_{1}^{k}\left(h_{2}^{k}\right)^{-1} & & & \\
& h_{2}^{k} g_{2}^{k}\left(h_{3}^{k}\right)^{-1} & & \\
& & h_{3}^{k} g_{3}^{k}\left(h_{4}^{k}\right)^{-1} & \\
& & \ddots & \\
& & & h_{L_{k}}^{k} g_{L_{k}}^{k}\left(h_{1}^{k}\right)^{-1}
\end{array}\right) c_{k} .
$$

For any element $h_{1}^{k} \in \Gamma$, one can choose

$$
h_{2}^{k}=h_{1}^{k} g_{1}^{k}, h_{3}^{k}=h_{2}^{k} g_{2}^{k}, \ldots, h_{L_{k}}^{k}=h_{L_{k}-1}^{k} g_{L_{k}-1}^{k}
$$

such that

$$
H_{k} D_{k} c_{k} H_{k}^{-1}=\left(\begin{array}{ccccc}
1 & & & & \\
& 1 & & & \\
& & 1 & & \\
& & \ddots & \\
& & & h_{1}^{k} g_{1}^{k} g_{2}^{k} \cdots g_{L_{k}}^{k}\left(h_{1}^{k}\right)^{-1}
\end{array}\right) c_{k}
$$


So, each conjugacy class of $\Gamma$ ? $S_{N}$ is described by the set of cycles in the decomposition (3.5), (3.6) of $\sigma_{0}$, where each cycle is marked by some conjugacy class of $\Gamma$.

The cycle of length $r$ marked by the conjugacy class $\alpha$ of $\Gamma$ with representative $g_{\alpha} \in \Gamma$ has the shape:

$$
A_{\alpha, r}:=\left(\begin{array}{ccccc}
1 & & & \\
& 1 & & \\
& 1 & & \\
& & \ldots & \\
& & & g_{\alpha}
\end{array}\right) \cdot c^{r}=\left(\begin{array}{cccccc}
0 & 1 & 0 & 0 & \ldots & 0 \\
0 & 0 & 1 & 0 & \ldots & 0 \\
0 & 0 & 0 & 1 & \ldots & 0 \\
\ldots & \ldots & \ldots & \ldots & \ldots & \ldots \\
0 & 0 & 0 & 0 & \ldots & 1 \\
g_{\alpha} & 0 & 0 & 0 & \ldots & 0
\end{array}\right),
$$

where $\alpha=1, \ldots, C(\Gamma)$ and $r=1,2, \ldots$; the matrix $A_{\alpha, r}$ and the cycle $c^{r}$ are the $r \times r$ block matrices, each block being a $2 \times 2$ matrix.

So, each element $g \in \Gamma \nmid S_{N}$ is conjugate to the element of the shape

$$
\left(\begin{array}{cccc}
A_{\alpha_{1}, r_{1}} & & & \\
& A_{\alpha_{2}, r_{2}} & & \\
& & \cdots & \\
& & & A_{\alpha_{s}, r_{s}}
\end{array}\right)
$$

It is convenient to describe the conjugacy class of $\Gamma$ ? $S_{N}$ with representative (3.8) by the set of nonnegative integers $p_{r}^{\alpha}$, where $r=1,2,3, \ldots$, and $\alpha=1, \ldots, C(\Gamma)$, such that

$$
\sum_{\alpha, r} r p_{r}^{\alpha}=N
$$

The value $p_{r}^{\alpha}$ for some conjugacy class is the number of cycles $A_{\alpha, r}$ of length $r$ in the decomposition (3.8) marked by the conjugacy class $\alpha$ of the group $\Gamma$.

Note that in [13] the notation $m_{r}(\alpha)$ is used instead of $p_{r}^{\alpha}$ we use in this paper.

The restriction (3.9) can be omitted and can serve as definition of $N$ for each set of the numbers $p_{r}^{\alpha}$.

The number of conjugacy classes in $\Gamma 2 S_{N}$ is equal to

$$
\left.C(\Gamma) S_{N}\right)=\sum_{p_{r}^{\alpha}: \sum_{\alpha, r} r p_{r}^{\alpha}=N} 1 .
$$

The generating function $c(\Gamma, x)$ of the number of conjugacy classes is defined as

$$
c(\Gamma, x):=\sum_{N=0}^{\infty} C\left(\Gamma \imath S_{N}\right) x^{N}
$$

and is equal to

$$
c(\Gamma, x)=\sum_{p_{r}^{\alpha}} x^{\Sigma_{\alpha, r} r p_{r}^{\alpha}}=\sum_{p_{r}^{\alpha}=0}^{\infty} \prod_{r=1}^{\infty} \prod_{\alpha=1}^{C(\Gamma)}\left(x^{r}\right)^{p_{r}^{\alpha}}=\prod_{r=1}^{\infty} \prod_{\alpha=1}^{C(\Gamma)} \frac{1}{1-x^{r}}=(\Psi(x))^{C(\Gamma)},
$$

where $\Psi(x)$ is the Euler function

$$
\Psi(x):=\prod_{r=1}^{\infty} \frac{1}{1-x^{r}}
$$




\subsection{Characteristic polynomials of conjugacy classes}

Before seeking the generating functions $t(\Gamma, x)$ and $s(\Gamma, x)$, let us find the characteristic polynomial of the conjugacy class $g$ of $\Gamma$ ? $S_{N}$ identified by the set $p_{r}^{\alpha}$.

Let $P_{M}(\lambda):=\operatorname{det}(M-\lambda)$ be the characteristic polynomial of the matrix $M$. Then it is easy to see that

$$
P_{A_{\alpha, r}}(\lambda)=\operatorname{det}\left(A_{\alpha, r}-\lambda\right)=\operatorname{det}\left(g_{\alpha}-\lambda^{r}\right)=P_{g_{\alpha}}\left(\lambda^{r}\right),
$$

where the marked cycle $A_{\alpha, r}$ is defined by (3.7).

Let $g \in \Gamma$ ? $S_{N}$ be defined by Eq. (3.8).

Now, it is easy to show that

$$
\begin{aligned}
P_{g}(\lambda)=\operatorname{det}(g-\lambda) & =\prod_{i=1}^{s} \operatorname{det}\left(A_{\alpha_{i}, r_{i}}-\lambda\right) \\
& =\prod_{\alpha, r: p_{r}^{\alpha} \geqslant 1} \operatorname{det}\left(A_{\alpha, r}-\lambda\right)^{p_{r}^{\alpha}} \\
& =\prod_{\alpha, r: p_{r}^{\alpha} \geqslant 1}\left(\operatorname{det}\left(g_{\alpha}-\lambda^{r}\right)\right)^{p_{r}^{\alpha}}
\end{aligned}
$$

if $g$ is a representative of the congugacy class in $\Gamma \nmid S_{N}$ corresponding to the set $p_{r}^{\alpha}$.

Definition 3.1. We call a conjugacy class $t$-admissible, if its representative $g \in \Gamma$ ? $S_{N}$ is such that $P_{g}(1) \neq 0$.

Definition 3.2. We call a conjugacy class s-admissible, if its representative $g \in \Gamma$ ? $S_{N}$ is such that $P_{g}(-1) \neq 0$.

Definition 3.3. We call a marked cycle $A_{\alpha, r}$, see Eq. (3.7), $t$-admissible, if $P_{A_{\alpha, r}}(1) \neq 0$.

Definition 3.4. We call a marked cycle $A_{\alpha, r}$, see Eq. (3.7), s-admissible, if $P_{A_{\alpha, r}}(-1) \neq 0$.

Equation (3.10) implies the following statements:

Proposition 3.1. The conjugacy class of $\Gamma$ ᄀ $S_{N}$ identified by the set $p_{r}^{\alpha}$ is $t$-admissible, if and only if the marked cycle $A_{\alpha, r}$ is $t$-admissible for any pair $\alpha, r$ such that $p_{r}^{\alpha} \neq 0$,

Proposition 3.2. The conjugacy class of $\Gamma$ ? $S_{N}$ identified by the set $p_{r}^{\alpha}$ is s-admissible, if and only if the marked cycle $A_{\alpha, r}$ is s-admissible for any pair $\alpha, r$ such that $p_{r}^{\alpha} \neq 0$,

Recall that $g_{\alpha} \in \Gamma \subset \operatorname{Sp}(2, \mathbb{C})$, where $\Gamma$ is a finite group. So $\operatorname{det} g_{\alpha}=1$ and the Jordan normal form of $g_{\alpha}$ is diagonal. This implies that if $g_{\alpha}$ has +1 in its spectrum, then $g_{\alpha}=1$ and if $g_{\alpha}$ has -1 in its spectrum, then $g_{\alpha}=-1$. These facts together with Eq. (3.10) imply, in their turn, the following two propositions:

Proposition 3.3. The conjugacy class of $\Gamma$ ? $S_{N}$ identified by the set $p_{r}^{\alpha}$ is $t$-admissible if and only if $g_{\alpha} \neq 1$ for all $\alpha, r$ with $p_{r}^{\alpha} \neq 0$.

Proposition 3.4. The conjugacy class of $\Gamma ? S_{N}$ identified by the set $p_{r}^{\alpha}$ is s-admissible if and only if for any pair $\alpha, r$ such that $p_{r}^{\alpha} \neq 0$, at least one of the next three conditions holds:

a) $g_{\alpha} \neq-1$ and $g_{\alpha} \neq 1$, 
b) $r$ is even and $g_{\alpha}=-1$,

c) $r$ is odd and $g_{\alpha}=1$.

Note that the three sets of pairs $(r, \alpha)$ defined by the cases a), b), c) in Proposition 3.4 have empty pair-wise intersections.

Definition 3.5. Let $t_{r}(\Gamma)$ for $r=1,2 \ldots$ be equal to the number of different $\alpha$ such that $A_{\alpha, r}$ is $t$-admissible.

Evidently,

$$
t_{r}(\Gamma)=C(\Gamma)-1
$$

Definition 3.6. Let $s_{r}(\Gamma)$ for $r=1,2 \ldots$ be equal to the number of different $\alpha$ such that $A_{\alpha, r}$ is $s$-admissible.

Evidently, if $\Gamma \ni-1$, then

$$
s_{r}(\Gamma)=C(\Gamma)-1
$$

and if $\Gamma \not \ngtr-1$, then

$$
s_{r}(\Gamma)= \begin{cases}C(\Gamma)-1, & \text { if } r \text { is even } \\ C(\Gamma), & \text { if } r \text { is odd }\end{cases}
$$

\section{Combinatorial problem}

Consider the following combinatorial problem (analogous problems are considered in [8]).

Suppose we have an unlimited supply of 1 -gram colored weights for each of $n_{1}$ different colors, an unlimited supply of 2-gram colored weights for each of $n_{2}$ different colors, an unlimited supply of 3-gram colored weights for each of $n_{3}$ different colors, and so on. Let $a_{n_{1}, \ldots, n_{k}, \ldots}^{N}$ be the number of opportunities to choose weights from our set of total mass $N$ grams.

The problem is to find generating function

$$
F_{n_{1}, \ldots, n_{k}, \ldots}(x):=\sum_{N=0}^{\infty} a_{n_{1}, \ldots, n_{k}, \ldots}^{N} x^{N} .
$$

This problem is exactly the problem we discussed earlier. Namely, now we say " $r$-gram weight" instead of cycle of length $r$, and "the number of different colors $n_{r}$ " instead of the number $t_{r}$ (3.11) or $s_{r}$ (3.12)-(3.13) of different $\alpha$.

\section{Proposition 4.1.}

$$
F_{n_{1}+m_{1}, n_{2}+m_{2}, \ldots, n_{k}+m_{k}, \ldots}(x)=F_{n_{1}, n_{2}, \ldots, n_{k}, \ldots}(x) \cdot F_{m_{1}, m_{2}, \ldots, m_{k}, \ldots}(x) .
$$

Proof. To prove this proposition, it suffices to note that

$$
a_{n_{1}+m_{1}, n_{2}+m_{2}, \ldots, n_{k}+m_{k}, \ldots}^{N}=\sum_{M=0}^{N} a_{n_{1}, n_{2}, \ldots, n_{k}, \ldots}^{M} \cdot a_{m_{1}, m_{2}, \ldots, m_{k}, \ldots}^{N-M} .
$$


Introduce the functions

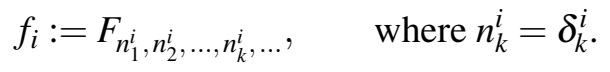

Then

$$
f_{i}(x)=1+x^{i}+x^{2 i}+x^{3 i}+\ldots=\frac{1}{1-x^{i}} .
$$

The next theorem follows from Proposition 4.1

\section{Theorem 4.1.}

$$
F_{n_{1}, n_{2}, \ldots, n_{k}, \ldots}=\prod_{i=1}^{\infty}\left(f_{i}\right)^{n_{i}} .
$$

The function $F_{1,1,1, \ldots}=\Psi(x)$ is the well-known Euler function, the generating function of the number of partitions of $N$ into the sum of positive integers.

\section{Generating functions $t(\Gamma)$ and $s(\Gamma)$}

Theorem 5.1. Set

$$
\begin{aligned}
& \Psi(x)=\prod_{i=1}^{\infty} \frac{1}{1-x^{i}} \quad \text { (Euler function), } \\
& \Phi(x):=\prod_{k=0}^{\infty} \frac{1}{1-x^{2 k+1}} .
\end{aligned}
$$

Let $T\left(\Gamma \geq S_{N}\right)$ be the dimension of the space of traces on $H_{1, \eta}\left(\Gamma \succ S_{N}\right)$ and let $S\left(\Gamma \succ S_{N}\right)$ be the dimension of the space of supertraces on $H_{1, \eta}\left(\Gamma<S_{N}\right)$ considered as a superalgebra.

Let

$$
\left.t(\Gamma, x):=\sum_{N=0}^{\infty} T(\Gamma) S_{N}\right) x^{N} \text { and } s(\Gamma, x):=\sum_{N=0}^{\infty} S\left(\Gamma 2 S_{N}\right) x^{N}
$$

Then

$$
\begin{aligned}
& t(\Gamma, x)=(\Psi(x))^{C(\Gamma)-1}, \\
& s(\Gamma, x)=(\Psi(x))^{C(\Gamma)-1}, \quad \text { if } \Gamma \neq \mathbf{Z}_{2 k+1}, \\
& s(\Gamma, x)=(\Psi(x))^{C(\Gamma)-1} \Phi(x), \text { if } \Gamma=\mathbf{Z}_{2 k+1} .
\end{aligned}
$$

Proof. To prove Theorem 5.1, we apply Theorem 4.1 to the numbers (3.11)-(3.13) of admissible conjugacy classes. It is clear that

$$
\begin{aligned}
& t(\Gamma)=F_{t_{1}(\Gamma), t_{2}(\Gamma), t_{3}(\Gamma), \ldots}=\Psi^{C(\Gamma)-1}, \\
& s(\Gamma)=F_{s_{1}(\Gamma), s_{2}(\Gamma), s_{3}(\Gamma), \ldots}= \begin{cases}\Psi^{C(\Gamma)-1}, & \text { if } \Gamma \ni-1, \\
\Psi^{C(\Gamma)-1} \Phi, & \text { if } \Gamma \not \supset-1 .\end{cases}
\end{aligned}
$$

Observe that $\Phi(x)=\sum_{i=0}^{\infty} O_{N} x_{N}$, where $O_{N}$ is the number of partitions of $N$ into the sum of odd positive integers, and $O_{N}$ coinsides with the number of independent supertraces on $H_{1 \eta}\left(S_{N}\right)$, see [12]. 


\subsection{Inequality theorem}

Theorem 5.2. Let $G=\Gamma \backslash S_{N}$. For each positive integer $N$, the following statements hold:

$$
\begin{aligned}
& S(G)>0, \\
& S(G) \geqslant T(G), \\
& S(G)=T(G) \text { if and only if } H_{1, \eta}(G) \text { contains a Klein operator. }
\end{aligned}
$$

Literally the same statements were proved for the groups $G$ from Family 1) in [10], and hence these statements hold for the direct product of any finite number of groups from Family 1) and Family 2) defined on page 1.

Proof. Let $\Gamma \neq \mathbf{Z}_{2 k+1}$. Since each finite group $\Gamma \in S p(2 N, \mathbb{C})$, except $\Gamma=\mathbf{Z}_{2 k+1}$, contains -1 , the group $\Gamma$ ? $S_{N}$ contains Klein operator $K=\prod_{i=1}^{N} D_{-1, i}$.

There is no Klein operator in $H_{1, \eta}\left(\mathbf{Z}_{2 k+1} \prec S_{N}\right)$ since for this algebra, $S\left(\mathbf{Z}_{2 k+1} \prec S_{N}\right)>T\left(\mathbf{Z}_{2 k+1}\right.$ থ $\left.S_{N}\right)$, as it follows from Theorem 5.1.

\section{Acknowledgments}

The authors (S.K. and I.T.) are grateful to Russian Fund for Basic Research (grant No. 17-02-00317) for partial support of this work.

\section{References}

[1] J. Bernstein, "Notes on supersymmetry". In: Deligne P., Etingof P., Freed D., Jeffrey L., Kazhdan D., Morgan J., Morrison D., Witten E. (eds.) Quantum fields and strings: a course for mathematicians. Vol. 1. Material from the Special Year on Quantum Field Theory held at the Institute for Advanced Study, Princeton, NJ, 1996-1997. American Mathematical Society, Providence, RI; Institute for Advanced Study (IAS), Princeton, NJ, 1999. Vol. 1: xxii+723 pp.

[2] K.A. Brown and I. Gordon, "Poisson orders, symplectic reflection algebras and representation theory", J. Reine Angew. Math. 559 (2003) 193-216.

[3] P. Etingof and V. Ginzburg, "Symplectic reflection algebras, Calogero-Moser space, and deformed Harish-Chandra homomorphism", Inv. Math. 147 (2002) 243-348.

[4] P. Etingof and S. Montarani, "Finite-dimensional representations of symplectic reflection algebras associated to wreath products", Representation Theory, An Electronic Journal of the American Mathematical Society Volume 9, Pages 457-467 (July 21, 2005) S 1088-4165(05)00288-8.

[5] I. Gordon, "Symplectic reflection algebras". BMC talk (2005), http://www.maths.ed.ac.uk/ igordon/BMC.pdf.

[6] I. Gordon, Symplectic reflection algebras. In: Trends in Representation Theory of Algebras and Related Topics, EMS Ser. Congr. Rep., Eur. Math. Soc., Zurich, pp. 285-347 (2008).

[7] R. Guralnick and J. Saxl, "Generation of finite almost simple groups by conjugates", J. Algebra 268 (2003), 519-571.

[8] M. Hall, Combinatorial theory, 2nd edition, Wisley, New York (1986), 464 pp.

S.K. Lando, Lectures on generating functions. Series: Student mathematical library, v. 23, American Mathematical Society in Providence, RI, (2003), 144 pp.

[9] C.W. Huffman and D. Wales, "Linear groups containing an element with an eigenspace of codimension two", in: Proceedings of the Conference on Finite Groups, Univ. Utah, Park City, Utah, 1975, Academic Press, New York, 1976, 425-429.

[10] S.E. Konstein and R. Stekolshchik, "Klein operator and the Number of Traces and Supertraces on the Superalgebra of Observables of Rational Calogero Model based on the Root System", Journal of Nonlinear Mathematical Physics 20 (2) (2013) 295-308. 
S.E. Konstein and I.V. Tyutin / The number of independent Traces and Supertraces on the Symplectic Reflection Algebra $H_{1, \eta}\left(\Gamma S_{N}\right)$

[11] S.E. Konstein, I.V. Tyutin, "The number of independent traces and supertraces on symplectic reflection algebras”, Journal of Nonlinear Mathematical Physics 21 (3) (2014) 308-335; arXiv:1308.3190.

[12] S.E. Konstein and M.A. Vasiliev, "Supertraces on the Algebras of Observables of the Rational Calogero Model with Harmonic Potential”, J. Math. Phys. 37 (1996) 2872.

[13] I.G. Macdonald, Symmetric functions and Hall polynomials - 2nd ed. (Oxford mathematical monographs), 1995, 475 pp., Appendix B, p. 169.

[14] D.S. Passman, Infinite Crossed Products, Pure and Applied Math vol. 135, Academic Press, San Diego, 1989.

[15] R. Stekolshchik, Notes on Coxeter Transformatios and the McKay Correspondence. Series: Springer Monographs in Mathematics 2008, XX, 239 pp. 